\title{
Strongly reduced penetration of atomic deuterium in radiation-damaged tungsten
}

\author{
M.H.J. 't Hoen, ${ }^{1, *}$ M. Mayer, ${ }^{2}$ A.W. Kleyn, ${ }^{1,3}$ and P.A. Zeijlmans van Emmichoven ${ }^{1}$ \\ ${ }^{1}$ FOM Institute DIFFER - Dutch Institute for Fundamental Energy Research, Association EURATOM-FOM, \\ Partner in the Trilateral Euregio Cluster, NL-3439 MN Nieuwegein, the Netherlands \\ ${ }^{2}$ Max-Planck-Institut für Plasmaphysik, Boltzmannstraße 2, D-85748 Garching, Germany \\ ${ }^{3}$ Van 't Hoff Institute for Molecular Sciences, University of Amsterdam, Science Park 904, \\ NL-1098 XH Amsterdam, The Netherlands, NL-1098 XH Amsterdam, the Netherlands
}

(Dated: November 5, 2013)

\begin{abstract}
Radiation-damaged tungsten is exposed to high-flux, low-energy deuterium plasmas at self-bias conditions. We observe that the fraction of deuterium that penetrates, is only $10^{-5}-10^{-7}$ of the plasma flux and strongly dependent on the local surface temperature. We propose that deuterium does not directly penetrate bulk tungsten but that it thermalizes at the surface where it forms a protective chemisorbed layer. We find an energy barrier of $1-2 \mathrm{eV}$ between surface and bulk, causing the influx of deuterium to be low as compared to the number of defects and leading to slow filling of the damaged layer.
\end{abstract}

PACS numbers: PACS 52.40.Hf, 52.77.Dq, 68.43.-h

Tungsten is foreseen as the main candidate material for use in the divertor of the experimental fusion reactor ITER. The divertor serves as the exhaust of the tokamak where the helium ash is removed. It is particular in this region where strong interaction of plasma with plasma facing components (PFC's) takes place. The plasma in the divertor region has a high electron density $\left(10^{20}-10^{21} \mathrm{~m}^{-3}\right)$ and a temperature of $1-10 \mathrm{eV}$. This leads to extremely intense particle and energy flux densities of $\sim 10^{24}$ ions $\mathrm{m}^{-2} \mathrm{~s}^{-1}$ and $\sim 10 \mathrm{MW} \mathrm{m}^{-2}$ respectively [1]. Its high thermal conductivity and melting point as well as its low erosion rate make tungsten favorable over many other materials. Another essential property of PFC's is that the uptake of tritium should be limited. For safety and efficiency reasons, the total tritium inventory in ITER should be kept below $700 \mathrm{~g}$ [2]. For tungsten it is known that the retention of hydrogen particles is low $\left(<10^{-4}\right.$ at.fr). However, radiation damage in a fusion reactor can lead to enhancement of the retention by orders of magnitude $[3,4]$.

In this letter we present deuterium retention studies for radiation damaged tungsten targets, exposed to atomic deuterium plasmas under ITER-like conditions. For ion temperatures of a few $\mathrm{eV}$ it is known that $90-95 \%$ of the deuterium is directly reflected $[5,6]$. However, the plasma flux is so high that there are large amounts of remaining deuterium atoms that interact with the tungsten surface. This results in a very complex plasmasurface interaction leading to adsorption of deuterium at the surface, recombination of atomic deuterium to molecules and release into the vacuum and/or back into the plasma $[7,8]$, penetration of deuterium in tungsten, etc. The situation is very schematically depicted in Fig. 1 with $\Phi_{\text {plasma }}$ the plasma flux and $\Phi_{\text {back }}$ the back flux containing directly reflected deuterium as well as thermalized deuterium leaving the surface as atoms and/or molecules. The effective ingoing flux, $\Phi_{\text {in }}$, is the fraction $f$ of the plasma flux that enters the tungsten material. In the present work we have monitored the diffusion of deuterium inwards from which we were able to directly determine $\Phi_{\text {in }}$ and thereby the fraction $f$. We obtain a value for $f$ that is very small, $10^{-5}-10^{-7}$. This fraction is strongly dependent on the local surface temperature and orders of magnitude lower than expected on the basis of the direct reflection mentioned above. We propose that this small value for $f$ originates in the deuterium not directly entering bulk tungsten but thermalising and neutralising at the surface forming a protective chemisorbed layer. Before diffusing into the bulk, the atoms need to cross an energy barrier that strongly limits the influx. We will show that an activation energy for crossing the barrier of $1-2 \mathrm{eV}$ is consistent with the temperature dependence of the influx.

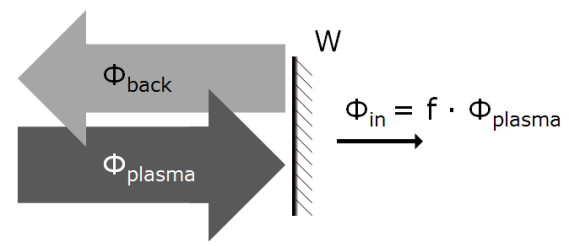

FIG. 1. The influx, $\Phi_{\text {in }}$, is only a small fraction $f$ of the incoming plasma flux, $\Phi_{\text {plasma }}$.

Polycrystalline tungsten targets (PLANSEE, 99.96\% purity, $\varnothing 20 \mathrm{~mm}, 1 \mathrm{~mm}$ thick) were mechanically polished until mirror finish and subsequently heated for one hour at $1273 \mathrm{~K}$ at a background pressure of $5 \times 10^{-4} \mathrm{~Pa}$. The targets were pre-irradiated at room temperature with $12.3 \mathrm{MeV} \mathrm{W}^{4+}$ ions at the $3 \mathrm{MV}$ ion accelerator at IPP Garching to homogeneous lateral damage. The fluence of the pre-irradiations was taken such that a damage level of 0.45 displacements per atom was reached (average displacement energy $90 \mathrm{eV}$ ). We have previously shown that this fluence is sufficient to saturate the damage level and 
that the damage extends to a depth of about $1.5 \mu \mathrm{m}$ below the surface [3]. The surface cleanliness was checked by X-ray photoelectron spectroscopy. Since the targets were stored under ambient conditions, they were covered with nanometer-thick native layers of oxygen and carbon $[3,9]$. During plasma exposure, most of these layers are removed within the first few seconds although the presence of in particular some oxygen cannot be excluded [10]. After plasma exposure, small amounts of Mo, Ca and $\mathrm{F}$ were found to be present on the surface. In most cases the concentrations were well below 10 at.\%. These impurities may slightly affect the protective layer and the energy barrier that we found in our measurements.

The pre-damaged tungsten targets were exposed to intense deuterium plasma beams at the linear plasma generator Pilot-PSI [11]. The plasma was created by a cascaded arc source and transported to the target by an axial magnetic field of $0.4 \mathrm{~T}$. During the exposures, the targets were water-cooled and electrically floating. Electron density and temperature profiles of the plasma beam were determined by Thomson scattering [12]. The plasma beam has approximately a Gaussian profile with maximum electron density and temperature of about $4 \times 10^{20} \mathrm{~m}^{-3}$ and $1 \mathrm{eV}$ respectively. The ion flux on the targets can be estimated by applying the Bohm criterion [13] and ranges from $2 \times 10^{24}$ ions $\mathrm{m}^{-2} \mathrm{~s}^{-1}$ in the center of the target to $5 \times 10^{23}$ ions $\mathrm{m}^{-2} \mathrm{~s}^{-1}$ at the edges. For our experimental conditions, the deuterium in the plasma predominantly consists of atomic ions $[14,15]$. The kinetic energy of the ions arriving at the surface can be estimated by considering a drifting Maxwellian velocity distribution for the ions in the plasma and an acceleration over the plasma sheath towards the surface. Assuming equal electron and ion temperatures in the plasma [15], this leads to an ion kinetic energy of roughly $5 \mathrm{eV}$. The temperature profile of the tungsten surface during exposure was measured with a fast infrared camera (FLIR SC7500-MB) and varies between $560 \mathrm{~K}$ in the center of the target to $470 \mathrm{~K}$ at the edges (the emissivity of the tungsten targets used was 0.07 , a value verified ex situ with the help of a thermocouple measurement). The absolute uncertainty in the temperatures is of order $25 \mathrm{~K}$ and is mainly caused by the uncertainty in the emissivity and the transmission of the infrared radiation through the vacuum window. The relative uncertainty in the temperatures is a few $\mathrm{K}$.

Deuterium depth profiles were measured one month after plasma exposure by nuclear reaction analysis (NRA) making use of the nuclear reaction $\mathrm{D}\left({ }^{3} \mathrm{He}, \mathrm{p}\right)^{4} \mathrm{He}$ [16]. The ${ }^{3} \mathrm{He}$ beam spot of about $1 \mathrm{~mm}$ in diameter was positioned at six spots on the target: two close to the center and two at $3 \mathrm{~mm}$ and two at $6 \mathrm{~mm}$ from the center at opposite sides. In this way, we obtain deuterium depth profiles for three different exposure temperatures, i.e., $560 \mathrm{~K}, 530 \mathrm{~K}$ and $500 \mathrm{~K}$. Differences between the measurements taken at the same temperature were small and only averages will be shown. At each position, the beam energy was scanned from $690 \mathrm{keV}$ to $4.0 \mathrm{MeV}$ to determine the deuterium concentration with a resolution of about $0.5 \mu \mathrm{m}$ down to a depth of $6 \mu \mathrm{m}$. The depth profiles of retained deuterium were calculated from the measured proton energy distributions with the NRA-DC program [17]. The total amount of deuterium in the top $6 \mu \mathrm{m}$ was obtained by integrating the depth profiles.

Five identical tungsten targets were exposed to deuterium plasma. The plasma fluxes and the corresponding surface temperatures were very similar in all experiments. The difference between the targets was the exposure time that was varied over almost two orders of magnitude, from $30 \mathrm{~s}$ to $2250 \mathrm{~s}$. This means a variation in fluence in the center of the targets from about $6 \times 10^{25} \mathrm{~m}^{-2}$ to $4.5 \times 10^{27} \mathrm{~m}^{-2}$ deuterium ions (fluence equals time integrated flux).

The NRA results for three of the targets are shown in Figs. 2a-c. For the one but shortest exposure time (Fig. 2a), the deuterium is retained mostly within $0.5 \mu \mathrm{m}$ from the surface for all three temperatures. At longer exposure times (Fig. 2b) it is clear that, in particular for the highest temperature, deuterium penetrates deeper in the material. This penetration continues for the longest exposure time as shown in Fig. 2c. The observations qualitatively agree with a process that is determined by diffusion: deeper penetration of deuterium for longer exposures and higher temperatures. For comparison, we also show the NRA results for a target that was exposed for $675 \mathrm{~s}$ to deuterium plasma at similar plasma conditions but biased at a potential of $-40 \mathrm{~V}$ (Fig. 2d). In contrast to the self-bias experiments, for all three temperatures the damaged slab has been filled with deuterium as result of direct penetration of tungsten. This clearly different result will be discussed in more detail later.

In Fig. 3, we show the integrated, total amounts of deuterium in the top $6 \mu \mathrm{m}$ for the self-bias experiments. It is clear that for the highest temperature of $560 \mathrm{~K}$, deuterium retention saturates after approximately $1000 \mathrm{~s}$. The deuterium inventory at saturation corresponds to the damaged layer (thickness about $1.5 \mu \mathrm{m}$ ) being completely filled with deuterium (1.2 at.\%). For $530 \mathrm{~K}$, it seems that saturation is reached for the longest exposure time of $2250 \mathrm{~s}$. For the lowest temperature of $500 \mathrm{~K}$, full saturation is never obtained.

The experimentally observed durations for complete saturation of the damaged layer with deuterium are long as compared to what would be expected on the basis of the large plasma flux and the diffusion of deuterium in tungsten. Using the Frauenfelder diffusion coefficient of deuterium in tungsten of $D_{F}=D_{0} \exp \left[-0.39 /\left(k_{\mathrm{B}} T\right)\right]$ with $D_{0}=2.9 \times 10^{-7} \mathrm{~m}^{2} \mathrm{~s}^{-1}[18]$, shows that for temperatures of $500-560 \mathrm{~K}$ deuterium diffuses in less than a second through the damaged layer. The damaged layer has an areal density of trapped deuterium of $\sim 10^{21} \mathrm{~m}^{-2}$. The plasma flux, after correction for the direct reflection of deuterium from tungsten, amounts to $\sim 10^{23} \mathrm{~m}^{-2} \mathrm{~s}^{-1}$. 

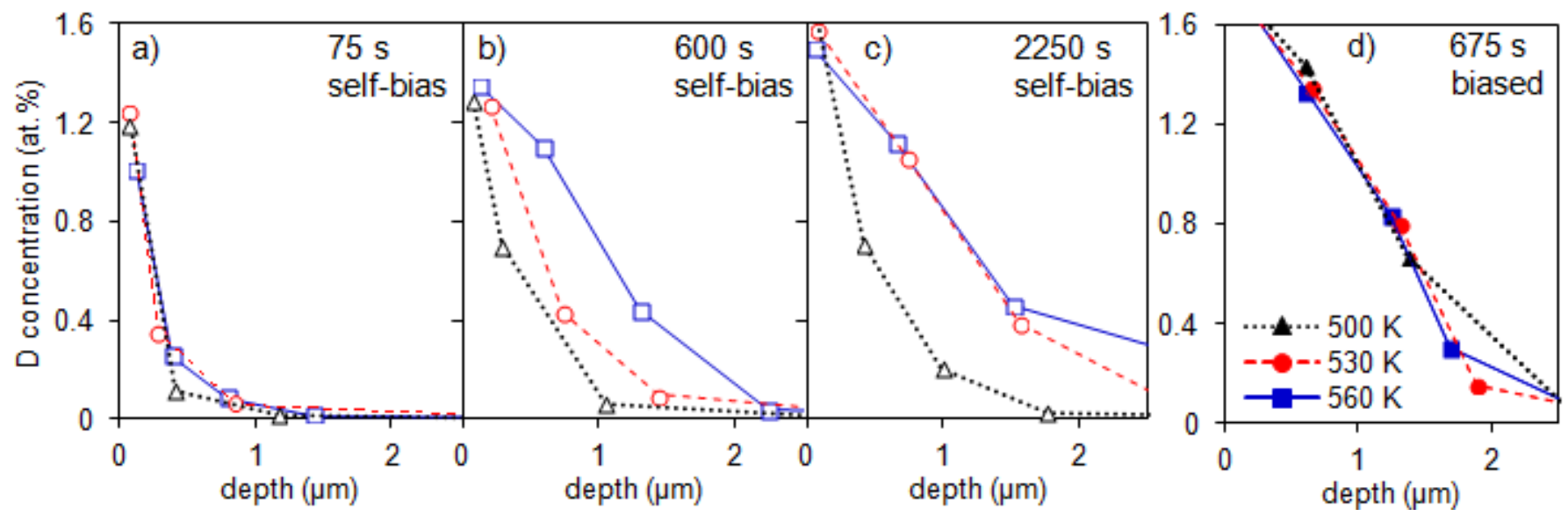

FIG. 2. Deuterium depth distributions in damaged (0.45 dpa) tungsten targets exposed to high-flux deuterium plasmas at self-bias conditions (a-c) and biased at $-40 \mathrm{~V}(\mathrm{~d})$. The exposure times are as indicated. The deuterium depth profiles were measured at three positions on the target (center at $560 \mathrm{~K}$, squares; $3 \mathrm{~mm}$ off-centre at $530 \mathrm{~K}$, circles; $6 \mathrm{~mm}$ off-centre at $500 \mathrm{~K}$, triangles)

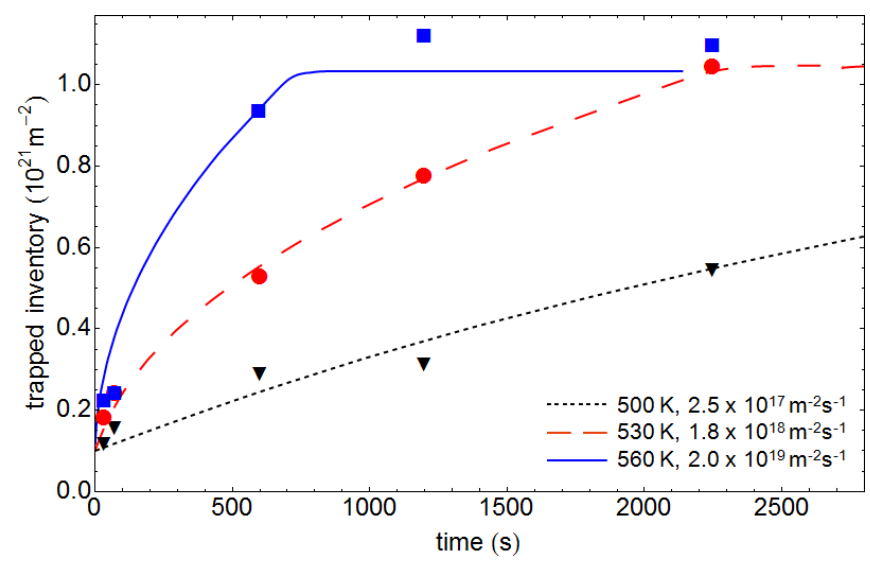

FIG. 3. The integrated, total amounts of deuterium in the top $6 \mu \mathrm{m}$ for the self-bias experiments. The lines show the results of the TMAP7 simulations.

This means that in one second plasma exposure there is ample deuterium available to fill the traps. This simple estimate thus yields a timescale that is more than three orders of magnitude faster than the experimentally observed saturation times. Uncertainties in the Frauenfelder diffusion coefficient [19] cannot explain this.

Why then is the experimentally observed filling of traps with deuterium three orders of magnitude slower than expected on the basis of plasma flux and diffusion? The assumption in the above considerations is that the high-flux plasma, after correction for reflection, directly penetrates into the outermost layers of the target. This seems to be a realistic assumption since the introduction of atomic deuterium in tungsten is known to be exothermic [20] and since the incoming ions in addition have a kinetic energy of $5 \mathrm{eV}$. However, our results strongly suggest that the assumption is not valid. We propose that instead of directly penetrating tungsten, the deuterium ions thermalize and neutralize at the surface where they form a chemisorbed layer. Presence of this layer in fact plays an important role in the thermalization process: deuterium-deuterium collisions cause energy pooling, thereby decreasing the energy of the incoming ions preventing them from direct penetration. This interpretation is supported by other experiments where it was shown that a chemisorbed deuterium layer on a barium surface actively decreases the interaction between incoming deuterium and barium atoms [21]. The contribution of the chemisorbed layer to the thermalization of deuterium means that it 'protects' tungsten from direct penetration by deuterium. To diffuse from this protective chemisorbed layer at the surface into bulk tungsten, deuterium needs to be activated over an energy barrier of a few eV [20,22]. This energy barrier reduces the effective influx of deuterium by many orders of magnitude to values so low that the filling of the damaged layer with deuterium is limited by the amount of traps and not by the diffusion coefficient. We will show that this interpretation leads to a consistent picture explaining the filling of the damaged layer being orders of magnitude slower than initially expected.

To determine values for $\Phi_{\text {in }}$ that are consistent with our experimental observations, we carried out TMAP7 simulations. TMAP7 is a one-dimensional program that solves the diffusion equation for deuterium in materials [23]. The program includes trapping of deuterium and recombination to molecules at the surface. Following our previous results [9], we assume three different trap sites for deuterium in tungsten with trap densities of $2 \times$ $10^{-1}$ at. $\%, 4.5 \times 10^{-1}$ at. $\%$, and $6.5 \times 10^{-1}$ at. $\%$ for trapping energies of $1.2 \mathrm{eV}, 1.4 \mathrm{eV}$, and $1.85 \mathrm{eV}$ respectively. For the diffusion coefficients of deuterium in tungsten, the above mentioned Frauenfelder values were used. For recombination of two atoms at the surface to a molecule, 


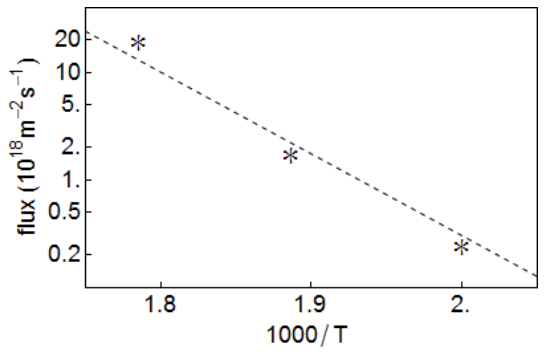

FIG. 4. The simulated influx as function of $1000 / T$ with $T$ the local target temperature.

a coefficient of $3.2 \times 10^{-15} \mathrm{~m}^{4} \mathrm{~s}^{-1} \exp \left[-1.16 /\left(k_{\mathrm{B}} T\right)\right]$ was taken [24]. The simulations were run with the flux varied until good agreement with the experiments was obtained. The results of the simulations are shown in Fig. 3. The deuterium influxes, $\Phi_{\text {in }}$, used were $2 \times 10^{19} \mathrm{~m}^{-2} \mathrm{~s}^{-1}(560 \mathrm{~K}), 1.8 \times 10^{18} \mathrm{~m}^{-2} \mathrm{~s}^{-1}(530 \mathrm{~K})$, and $2.5 \times 10^{17} \mathrm{~m}^{-2} \mathrm{~s}^{-1}(500 \mathrm{~K})$, which means that $f$ is in the range of $10^{-5}$ to $10^{-7}$. Note that $\Phi_{\text {in }}$ is the deuterium influx in the first nanometer of the material. Part of this influx leaves the surface again via recombination. To fit the experimental data, the simulated curves have slightly been shifted upwards by $1 \times 10^{20} \mathrm{~m}^{-2}$. The kinks in the simulated curves for the two highest temperatures at $750 \mathrm{~s}$ and $2200 \mathrm{~s}$ correspond to the times where the damaged layer is filled with deuterium. The simulations reproduce the experiments well. The effective influx turns out to be strongly dependent on temperature: almost two orders of magnitude variation is obtained in the temperature range of only $60 \mathrm{~K}$. This dependence is much stronger than the variation in plasma flux, which varies only by about $30 \%$.

In Fig. 4, the simulated influx is plotted as function of the inverse temperature. The datapoints follow quite well a straight line showing that the process has Arrheniustype behavior $\left(\Phi_{\text {in }}=\Phi_{\text {in }}\left(T_{\infty}\right) \exp \left[-E_{a} / k_{\mathrm{B}} T\right]\right)$ with $\Phi_{\text {in }}$ the influx of deuterium, $k_{\mathrm{B}}$ Boltzmann's constant and $T$ the local temperature of the surface during plasma exposure. The activation energy $E_{a}$ directly follows from the slope and amounts to $1.6 \mathrm{eV}$. The pre-exponential factor, $\Phi_{\text {in }}\left(T_{\infty}\right)$, represents the surface coverage of chemisorbed deuterium, $\left(1-2 \times 10^{19} \mathrm{~m}^{-2}\right)[25]$ multiplied by the attempt frequency, typically in the order of $10^{13} \mathrm{~s}^{-1}$. We fixed this value at $10^{32} \mathrm{~m}^{-2} \mathrm{~s}^{-1}$. Taking into account the uncertainties in the experimental values for the temperature, the results become $E_{a}=(1-2) \mathrm{eV} . E_{a}$ is close to reported energy barriers of around $2 \mathrm{eV}$ for diffusion of chemisorbed deuterium from surface to bulk [20,22].

In conclusion, we have shown that the experimentally observed, slow diffusion of deuterium in pre-irradiated tungsten is consistent with the deuterium atomic ions not directly penetrating in bulk tungsten but forming a chemisorbed layer at the surface. This leads to a reduction in the effective influx of deuterium by many orders of magnitude as compared to the plasma flux. Obviously, at self-bias conditions, the kinetic energy of the atomic ions arriving at the surface of roughly $5 \mathrm{eV}$ is not sufficient for penetration of the surface with its protective layer. For a target biased at a potential of $-40 \mathrm{~V}$, on the other hand, we observed that the damaged layer is filled on a much shorter timescale. This suggests that, upon their acceleration towards the surface by the bias potential, the ions directly penetrate the bulk leading to high concentrations of deuterium and fast filling of the damaged layer. Our results are to some extent reminiscent of what has been observed by others on the diffusion of deuterium in tungsten containing considerable amounts of defects ([26]. Also in these experiments the trapping of deuterium per unit time was the limiting factor for diffusion. However, the kinetic energy of the deuterium ions was so high that direct penetration occurred. Our observations are furthermore qualitatively consistent with the molecular dynamics simulations of [5], where it was shown that individual hydrogen atoms incident on perfect tungsten at kinetic energies up to $3 \mathrm{eV}$ either reflect from or stick at the surface whereas at $10 \mathrm{eV}$ a small fraction directly penetrates. In a comparison with these simulations, one should realize that our high-flux plasma leads to the presence of large amounts of deuterium in the neighborhood of the surface including a chemisorbed layer. As discussed this chemisorbed layer can act as a protective layer preventing direct penetration. Finally, our results shed light on previously reported strong surface modifications of tungsten during exposure to deuterium plasmas at biased conditions [27, 28] as opposed to the absence of such modifications for self-bias conditions. The surface modifications can be related to an oversaturation of tungsten with deuterium originating in the direct penetration. The absence of surface modifications at self-bias conditions can readily be understood in terms of the absence of direct penetration, as was shown in the present letter.

This work, supported by the European Communities under the contract of Association between EURATOM/FOM, was carried out within the framework of the European Fusion Programme with financial support from NWO. The views and opinions expressed herein do not necessarily reflect those of the European Commission. The authors would like to thank R.S. Al for the technical assistance at the Pilot-PSI measurements, J. Dorner and M. Fußeder for their technical assistance with the ${ }^{3} \mathrm{He}$ beam analysis and E. Zoethout for the XPS analysis of the samples.

*m.h.j.thoen@differ.nl

[1] G. Federici, C. H. Skinner, J. N. Brooks, J. P. Coad, C. Grisolia, A. A. Haasz, A. Hassanein, V. Philipps, C. S. 
Pitcher, J. Roth, W. R. Wampler, and D. G. Whyte, Nuclear Fusion 41, 1967 (2001).

[2] J. Roth, E. Tsitrone, A. Loarte, T. Loarer, G. Counsell, R. Neu, V. Philipps, S. Brezinsek, M. Lehnen, P. Coad, C. Grisolia, K. Schmid, K. Krieger, A. Kallenbach, B. Lipschultz, R. Doerner, R. Causey, V. Alimov, W. Shu, O. Ogorodnikova, A. Kirschner, G. Federici, and A. Kukushkin, Journal of Nuclear Materials 390-391, 1 (2009).

[3] M. H. J. 't Hoen, B. Tyburska-Pueschel, K. Ertl, M. Mayer, J. Rapp, A. W. Kleyn, and P. A. Zeijlmans van Emmichoven, Nuclear Fusion 52, 023008 (2012).

[4] M. Shimada, G. Cao, Y. Hatano, T. Oda, Y. Oya, M. Hara, and P. Calderoni, Physica Scripta 2011, 014051 (2011).

[5] K. O. E. Henriksson, K. Voertler, S. Dreißigacker, K. Nordlund, and J. Keinonen, Surface Science 600, 3167 (2006).

[6] S. Markelj, O. V. Ogorodnikova, P. Pelicon, T. SchwarzSelinger, and I. Cadež, Applied Surface Science, (2013).

[7] R. Gat and J. C. Angus, Journal of Applied Physics 74, 5981 (1993).

[8] M. Rutigliano and M. Cacciatore, Phys. Chem. Chem. Phys. 13, 7475 (2011).

[9] M. H. J. 't Hoen, M. Mayer, A. W. Kleyn, H. Schut, and P. A. Zeijlmans van Emmichoven, Nuclear Fusion 53, 043003 (2013).

[10] S. Xu and L. Diao, Journal of Vacuum Science \& Technology A: Vacuum, Surfaces, and Films 26, 360 (2008).

[11] G. J. van Rooij, V. P. Veremiyenko, W. J. Goedheer, B. de Groot, A. W. Kleyn, P. H. M. Smeets, T. W. Versloot, D. G. Whyte, R. Engeln, D. C. Schram, and N. J. L. Cardozo, Applied Physics Letters 90, 121501 (2007).

[12] H. J. van der Meiden, R. S. Al, C. J. Barth, A. J. H. Donné, R. Engeln, W. J. Goedheer, B. de Groot, A. W. Kleyn, W. R. Koppers, N. J. L. Cardozo, M. J. van de Pol, P. R. Prins, D. C. Schram, A. E. Shumack, P. H. M. Smeets, W. A. J. Vijvers, J. Westerhout, G. M. Wright, and G. J. van Rooij, Review of Scientific Instruments 79, 013505 (2008).

[13] D. Bohm, The Characteristics of Electrical Discharges in Magnetic Fields, chapter 3, edited by A. Guthrie and
R. K. Wakerling (NewYork: McGraw-Hill, 1949).

[14] R. C. Wieggers, P. W. C. Groen, H. J. de Blank, and W. J. Goedheer, Contributions to Plasma Physics 52, 440 (2012).

[15] A. E. Shumack, V. P. Veremiyenko, D. C. Schram, H. J. de Blank, W. J. Goedheer, H. J. van der Meiden, W. A. J. Vijvers, J. Westerhout, N. J. Lopes Cardozo, and G. J. van Rooij, Phys. Rev. E 78, 046405 (2008).

[16] M. Mayer, E. Gauthier, K. Sugiyama, and U. von Toussaint, Nuclear Instruments and Methods in Physics Research Section B: Beam Interactions with Materials and Atoms 267, 506 (2009).

[17] K. Schmid and U. von Toussaint, Nuclear Instruments and Methods in Physics Research Section B: Beam Interactions with Materials and Atoms 281, 64 (2012).

[18] R. Frauenfelder, Journal of Vacuum Science and Technology 6, 388 (1969).

[19] R. A. Causey, Journal of Nuclear Materials 300, 91 (2002).

[20] A. V. Veen, H. A. Filius, J. D. Vries, K. R. Bijkerk, G. J. Rozing, and D. Segers, Journal of Nuclear Materials 155-157, 1113 (1988).

[21] R. M. A. Heeren, D. Ćirić, H. J. Hopman, and A. W. Kleyn, Applied Physics Letters 59, 158 (1991).

[22] D. F. Johnson and E. A. Carter, Journal of Materials Research 25, 315 (2010).

[23] G. R. Longhurst, TMAPr User Manual, Tech. Rep. (INEEL/EXT-04-02352 (Idaho: Idaho National Engineering and Environmental Laboratory), 2004).

[24] R. A. Anderl, D. F. Holland, G. R. Longhurst, R. J. Pawelko, C. L. Trybus, and C. H. Sellers, Fusion Technology 21, 745 (1992).

[25] B. Feuerbacher and B. Fitton, Phys. Rev. B 8, 4890 (1973).

[26] R. A. Causey, R. Doerner, H. Fraser, R. D. Kolasinski, J. Smugeresky, K. Umstadter, and R. Williams, Journal of Nuclear Materials 390-391, 717 (2009).

[27] A. Manhard, K. Schmid, M. Balden, and W. Jacob, Journal of Nuclear Materials 415, S632 (2011).

[28] H. Xu, Y. Zhang, Y. Yuan, B. Fu, A. Godfrey, G. D. Temmerman, W. Liu, and X. Huang, Journal of Nuclear Materials 443, 452 (2013). 